\title{
Global existence for the discrete diffusive coagulation-fragmentation equations in $L^{1}$
}

\section{Philippe Laurençot and Stéphane Mischler}

\begin{abstract}
Existence of global weak solutions to the discrete coagulationfragmentation equations with diffusion is proved under general assumptions on the coagulation and fragmentation coefficients. Unlike previous works requiring $L^{\infty}$-estimates, an $L^{1}$-approach is developed here which relies on weak and strong compactness methods in $L^{1}$.
\end{abstract}

\section{Introduction}

Coagulation and fragmentation processes occur in the dynamics of cluster growth and describe the mechanisms allowing clusters to coalesce to form larger clusters or break apart into smaller ones. Since the pioneering works of Smoluchowski [21, 22] mean-field models have been developed to describe the dynamics of such processes, in which the clusters are assumed to be fully identified by their size (or volume or number of particles) which is either a positive real number (continuous model) or a positive integer (discrete model). The mean-field approach initiated by Smoluchowski intends to describe the evolution with respect to space and time of the cluster size distribution as the system of clusters undergoes various physical influences. Let us mention here that the original model proposed by Smoluchowski was introduced to analyze the binary coagulation of colloids moving according to brownian motions and several additional physical processes have been subsequently incorporated into the model (fragmentation, condensation, influence of external fields, see, e.g., [10]). The field of applications of the Smoluchowski model has meanwhile widened and now includes, among others,

2000 Mathematics Subject Classification: 35K50, 35K57, 82D60.

Keywords: Cluster growth, coalescence, breakage, infinite system of reaction-diffusion equations, existence, weak compactness. 
aerosol science (evolution of a system of solid or liquid particles suspended in a gas $[10,19]$ ), astrophysics (formation of the planets [18]), polymer science and hematology (red blood cell aggregation [17]). We refer to [10] for derivations of the model, together with some physical background.

In the model studied in this paper we assume that the size of the clusters is a discrete parameter ranging in the set of the positive integers and restrict ourselves to the following physical situation : the only reactions taken into account are the binary coagulation of clusters to form a larger one and the spontaneous fragmentation of clusters into smaller pieces (multiple fragmentation), while the approach of two clusters leading to aggregation is assumed to result only from brownian movement or diffusion (thermal coagulation). Other effects such as multiple coagulation or condensation, together with the influence of other external force fields (such as electric fields for charged particles) are neglected. Under these assumptions the discrete diffusive coagulation-fragmentation equations read

$$
\begin{aligned}
\partial_{t} c_{i}-d_{i} \Delta_{x} c_{i} & =Q_{i}(c) & & \text { in }(0,+\infty) \times \Omega, \\
\partial_{n} c_{i}= & 0 & & \text { on }(0,+\infty) \times \partial \Omega, \\
c_{i}(0)= & c_{i}^{i n} & & \text { in } \Omega,
\end{aligned}
$$

for $i \geq 1$, where $c_{i}=c_{i}(t, x) \geq 0$ denotes the local concentration of clusters of size $i$ (or $i$-clusters), $c=\left(c_{i}\right)_{i \geq 1}$ and

$$
Q_{i}(c)=Q_{1, i}(c)-Q_{2, i}(c)-Q_{3, i}(c)+Q_{4, i}(c), \quad i \geq 1,
$$

with

$$
\begin{aligned}
& Q_{1, i}(c)=\frac{1}{2} \sum_{j=1}^{i-1} a_{i-j, j} c_{i-j} c_{j}, \quad Q_{2, i}(c)=B_{i} c_{i}, \\
& Q_{3, i}(c)=L_{i}(c) c_{i} \quad \text { with } L_{i}(c)=\sum_{j=1}^{\infty} a_{i, j} c_{j}, \\
& Q_{4, i}(c)=\sum_{j=1}^{\infty} B_{i+j} \beta_{i+j, i} c_{i+j} .
\end{aligned}
$$

Here $\Omega$ is an open bounded subset of $\mathbb{R}^{d}, d \geq 1$, with smooth boundary $\partial \Omega$, $\partial_{n} c_{i}$ denotes the outward normal derivative of $c_{i}, d_{i}$ the diffusion coefficient of $i$-clusters while the kinetic coefficients $a_{i, j}, B_{i}$ and $\beta_{i, j}$ denote the coagulation rate of $i$-clusters with $j$-clusters $\left(a_{i, j}=a_{j, i}\right)$, the rate of fragmentation of $i$-clusters (with $B_{1}=0$ ) and the average number of $j$-clusters produced 
during the break-up of a $i$-cluster, respectively. Observe that conservation of mass during the fragmentation reaction implies

$$
\sum_{j=1}^{i-1} j \beta_{i, j}=i, \quad i \geq 2 .
$$

We finally indicate the quantitative meaning of the reaction terms in $Q_{i}(c)$ : $Q_{1, i}(c)$ accounts for the formation of $i$-clusters by coalescence of smaller clusters and $Q_{2, i}(c)$ for the breakage of $i$-clusters into smaller pieces. The term $Q_{3, i}(c)$ describes the depletion of $i$-clusters by coagulation with other clusters, while $Q_{4, i}(c)$ represents the gain of $i$-clusters resulting from the fragmentation of larger clusters. Notice that there are no sources nor sinks of clusters in the reactions described by (1)-(3), so that the total mass of clusters defined by

$$
\varrho(t)=\sum_{i=1}^{\infty} i \int_{\Omega} c_{i}(t, x) d x
$$

is expected to be constant throughout time evolution provided it is initially finite. It is however well-known by now that this property may fail to be true for some physically relevant kinetic coefficients, a phenomenon known as gelation. We will however not consider this issue here and refer to [13] and the references therein for results in that direction in the spatially homogeneous case. In the spatially inhomogeneous case, a formal description of the spatial behaviour near a gelation point is provided in [12] when $B_{i}=0$, $a_{i, j}=i j$ and $d_{i}=1$ (see also [20]). In general, the only piece of information within easy reach is that the total mass at time $t$ does not exceed the initial total mass, that is,

$$
\sum_{i=1}^{\infty} i \int_{\Omega} c_{i}(t, x) d x \leq \sum_{i=1}^{\infty} i \int_{\Omega} c_{i}^{i n}(x) d x .
$$

Still, the inequality (5) provides a natural functional framework to work within, and a first a priori estimate as well.

It is actually the aim of this paper to show that, under rather general assumptions on the kinetic coefficients, there exists at least a weak solution to (1)-(3) for any initial datum $c^{i n}=\left(c_{i}^{i n}\right)_{i \geq 1}$ with finite total mass. More precisely we assume that

$$
\begin{gathered}
0 \leq a_{i, j}=a_{j, i}, \quad i, j \geq 1 \\
B_{1}=0 \quad \text { and } \quad B_{i} \geq 0, \quad i \geq 2, \\
\left(\beta_{i, j}\right) \in[0,+\infty)^{i-1} \text { and } \sum_{j=1}^{i-1} j \beta_{i, j}=i, \quad i \geq 2,
\end{gathered}
$$


and the additional growth assumption

$$
\lim _{j \rightarrow+\infty} \frac{a_{i, j}}{j}=\lim _{j \rightarrow+\infty} \frac{B_{i+j} \beta_{i+j, i}}{i+j}=0, \quad i \geq 1 .
$$

While (6)-(8) are structural assumptions on the kinetic coefficients the growth condition (9) is more restrictive and is needed to control the tail of the series in $Q_{i}(c)$ with (5). Though it excludes some interesting cases such as $a_{i, j}=i j$ or $a_{i, j}=\left(i^{\alpha}+j^{\alpha}\right)^{1 / \alpha}, \alpha \in(0,1]$, it still includes a large number of physically relevant cases such as $a_{i, j}=i^{\alpha} j^{\alpha}, \alpha \in[0,1)$ or $a_{i, j}=\left(i^{\alpha}+j^{\alpha}\right)^{1 / \beta}$, $0 \leq \alpha<\beta \leq 1$. As for the fragmentation coefficients typical examples are

$$
B_{i}=i^{1+\mu} \text { and } \beta_{i, j}=i j^{\nu}\left(\sum_{k=1}^{i-1} k^{1+\nu}\right)^{-1}, \quad 1 \leq j<i,
$$

for $\mu \in \mathbb{R}$ and $\nu \in(-2,0]$ (see, e.g., [16]), and the growth condition (9) is fulfilled whenever $\mu<1+\nu$.

Remark 1 Our assumptions on the fragmentation coefficients clearly allow the breakage of clusters into more than two pieces. An important subclass of fragmentation coefficients is the so-called binary fragmentation case where only two clusters are produced in each fragmentation event. In that case, denoting by $b_{i, j}$ the rate of fragmentation of $(i+j)$-clusters into a $i$-cluster and a $j$-cluster $\left(b_{i, j}=b_{j, i}\right)$, the coefficients $B_{i}$ and $\beta_{i, j}$ are given by

$$
B_{i}=\frac{1}{2} \sum_{j=1}^{i-1} b_{j, i-j}, \quad B_{i} \beta_{i, j}=b_{j, i-j}, \quad 1 \leq j<i .
$$

As for the diffusion coefficients we only assume that

$$
d_{i}>0, \quad i \geq 1
$$

We also introduce the space $X_{1}$ of sequences of integrable functions with finite total mass, namely,

$$
X_{1}=\left\{c=\left(c_{i}\right)_{i \geq 1}, c_{i} \in L^{1}(\Omega), \sum_{i=1}^{\infty} i\left|c_{i}\right|_{L^{1}}<\infty\right\},
$$

which is a Banach space endowed with the norm

$$
\|c\|_{1}=\sum_{i=1}^{\infty} i\left|c_{i}\right|_{L^{1}}, \quad c \in X_{1} .
$$


The $c_{i}$ 's being local concentrations we will actually work in the positive cone $X_{1}^{+}$of $X_{1}$,

$$
X_{1}^{+}=\left\{c=\left(c_{i}\right)_{i \geq 1} \in X_{1}, \quad c_{i} \geq 0 \text { a.e. for } i \geq 1\right\} .
$$

We finally assume that the initial datum $c^{\text {in }}$ satisfies

$$
c^{i n}=\left(c_{i}^{i n}\right)_{i \geq 1} \in X_{1}^{+} .
$$

We may now define the notion of weak solution to (1)-(3) we will use throughout the paper.

Definition 1 A global weak solution $c=\left(c_{i}\right)_{i \geq 1}$ to (1)-(3) is a mapping from $[0,+\infty)$ to $X_{1}^{+}$such that for each $T \in(0,+\infty)$ and $i \geq 1$ there holds:

$$
\begin{gathered}
c_{i} \in \mathcal{C}\left([0, T] ; L^{1}(\Omega)\right), \\
\sum_{j=1}^{\infty} a_{i, j} c_{i} c_{j} \in L^{1}((0, T) \times \Omega), \\
\sup _{t \in[0,+\infty)}\|c(t)\|_{1} \leq\left\|c^{i n}\right\|_{1},
\end{gathered}
$$

and $c_{i}$ is a mild solution to the $i$-th equation of (1), i.e. $c_{i}$ satisfies for each $t \in[0,+\infty)$,

$$
c_{i}(t)=e^{d_{i} A_{1} t} c_{i}^{i n}+\int_{0}^{t} e^{d_{i} A_{1}(t-s)} Q_{i}(c(s)) d s,
$$

where $Q_{i}(c)$ is defined by (4), $A_{1}$ denotes the closure in $L^{1}(\Omega)$ of the unbounded linear operator $A$ of $L^{2}(\Omega)$ defined by

$$
D(A)=\left\{w \in H^{2}(\Omega), \quad \partial_{n} w=0 \quad \text { on } \partial \Omega\right\}, \quad A w=\Delta_{x} w,
$$

and $e^{d_{i} A_{1} t}$ is the $C_{0}$-semigroup generated by $d_{i} A_{1}$ in $L^{1}(\Omega)$.

Observe that, for each $i \geq 1,(13)$ guarantees that $Q_{1, i}(c)$ and $Q_{3, i}(c)$ are in $L^{1}((0, T) \times \Omega)$ while (8), (9) and (14) ensure that $Q_{2, i}(c)$ and $Q_{4, i}(c)$ belong to $L^{1}((0, T) \times \Omega)$; thus the integral formulation (15) makes sense. Notice also that the integrability property (13) of $Q_{3, i}(c)$ cannot be deduced from (14) and this point is actually the main difficulty encountered in the study of the existence of solutions to (1)-(3) in the spatially inhomogeneous case (such a difficulty is not met in the spatially homogeneous case). Since (14) seems to be the only a priori estimate available in the general case, each function $c_{i}$ is only integrable and the first issue to be considered is to 
find a functional setting in which the product $c_{i} c_{j}$ has suitable functional properties. A first method to handle this difficulty is to obtain $L^{\infty}$-estimates on the $c_{i}$ 's (possibly depending on $i$ ) and this approach has been used to prove most of the global existence results obtained so far for the general discrete coagulation-fragmentation equations with diffusion [6, 8, 11, 24, 25]. Such an approach however requires stringent restrictions on the kinetic coefficients and each component of the initial datum has to belong to $L^{\infty}(\Omega)$ as well. In particular, in order to allow unbounded coagulation and fragmentation coefficients, it is necessary to assume the fragmentation to be suitably dominated by the coagulation [24] or to assume the initial datum to be sufficiently small [7]. Weaker assumptions can be made in some special cases such as the pure coagulation case $\left(B_{i}=0,[6]\right)$, the pure fragmentation case $\left(a_{i, j}=0,[14]\right)$ or the Becker-Döring case [15], where the specific structure of these cases yields additional a priori estimates. Another approach has been recently introduced by Amann [2] which consists in viewing (1)-(3) as a single semilinear evolution equation for the Banach-space-valued function $c$ to which abstract results may be applied. Though this approach requires also the strong assumption of the boundedness of the kinetic coefficients together with some additional regularity with respect to space of the initial datum, it allows to handle general second-order operators and provides the existence, uniqueness and continuous dependence with respect to the initial data of solutions to (1)-(3), but only locally in time in the general case (global existence is obtained only in one-space dimension).

Since we are interested in global existence results we propose in this paper a third approach relying on weak compactness methods in $L^{1}(\Omega)$ which allows us to prove the existence of weak solutions to (1)-(3) in the general framework described above. More precisely, our result reads as follows :

Theorem 2 Assume that the kinetic coefficients $\left(a_{i, j}\right),\left(B_{i}\right),\left(\beta_{i, j}\right)$, the diffusion coefficients $\left(d_{i}\right)$ and the initial datum $c^{\text {in }}$ satisfy the assumptions (6)(9), (10) and (11), respectively. Then there is at least one global weak solution to (1)-(3).

The solutions to (1)-(3) constructed in Theorem 2 are of course less regular than the ones obtained in the above mentioned papers and we do not know whether there are unique or not. They however enjoy a weak stability principle, see Theorem 3 below. We however emphasize here again that no regularity assumptions are made on the initial datum except the natural one (11) and the growths of the coagulation and fragmentation coefficients do not depend on one another and have only to comply with (9). Let us finally recall that the existence theory is far more complete in the spatially homogeneous case (see, e.g., [3, 9, 23] and the references therein). 
We now briefly outline the proof of Theorem 2. We will in fact establish a weak stability property of weak solutions to (1)-(3) in the sense of Definition 1 (Theorem 3), that is, given a sequence of approximating solutions to (1)-(3) satisfying (14) uniformly, there is a subsequence which converges towards a weak solution to (1)-(3). More precisely, let $T \in(0,+\infty)$ and assume that $\left(c^{N}\right)_{N \geq 1}$ is a sequence of weak solutions to (1)-(3) such that $\left(c_{i}^{N}(0)\right)_{N}$ is weakly compact in $L^{1}(\Omega)$ for each $i \geq 1$ and

$$
\sup _{N \geq 1} \sup _{t \in[0, T)}\left\|c^{N}(t)\right\|_{1}<\infty
$$

First, by (9) and (16), the fragmentation terms are bounded in $L^{1}\left(\Omega_{T}\right)$ with $\Omega_{T}:=(0, T) \times \Omega$. The boundedness in $L^{1}\left(\Omega_{T}\right)$ of the coagulation terms then follows from (1) by induction on $i \geq 1$ and the elementary observations that $Q_{1,1}\left(c^{N}\right)=0$ and

$$
Q_{1, i}\left(c^{N}\right) \leq \sum_{j=1}^{i-1} Q_{3, j}\left(c^{N}\right), \quad i \geq 1
$$

The compactness properties of the heat equation next warrant that there are a subsequence of $\left(c^{N}\right)_{N}$ and $c=\left(c_{i}\right)$ such that $\left(c_{i}^{N}\right)_{N}$ converges towards $c_{i}$ in $L^{1}\left(\Omega_{T}\right)$ for each $i \geq 1$, which, together with (9), yield the strong compactness of the fragmentation terms in $L^{1}\left(\Omega_{T}\right)$. To prove the compactness of the coagulation terms, we proceed again by induction on $i \geq 1$. The key point is to observe that, if $\left(u_{k}\right)$ is a weakly compact sequence of non-negative functions in $L^{1}\left(\Omega_{T}\right)$ satisfying

$$
\partial_{t} u_{k}-d_{i} \Delta_{x} u_{k}+u_{k} g_{k} \leq f_{k} \quad \text { in } \Omega_{T}
$$

for some weakly compact sequences $\left(f_{k}\right)$ and $\left(g_{k}\right)$ of non-negative functions in $L^{1}\left(\Omega_{T}\right)$, the sequence $\left(u_{k} g_{k}\right)$ is also weakly compact in $L^{1}\left(\Omega_{T}\right)$. Applying this result successively to each equation of (1) yields the weak compactness of the coagulation terms. The strong compactness of the coagulation terms is next obtained with the help of (9) and the strong compactness of $\left(c_{i}^{N}\right)_{N}$ for each $i \geq 1$. The fact that $c$ is a weak solution to (1)-(3) then readily follows. Actually, the method of proof we just sketch also applies to sequences of solutions to suitable approximations to (1)-(3).

This paper is organized in the following way : the next section is devoted to the weak stability principle and the proof of Theorem 2 is given in Section 3. As in previous works on similar equations, the existence of weak solutions to (1)-(3) follows by taking the limit of solutions to approximating systems of reaction-diffusion equations obtained by suitable truncations of (1). From now on, we fix kinetic coefficients $\left(a_{i, j}\right),\left(B_{i}\right)$ and $\left(\beta_{i, j}\right)$ satisfying (6), (7) and (8), respectively, and (9) as well. We also fix a sequence of diffusion coefficients $\left(d_{i}\right)$ satisfying $(10)$. 


\section{Weak stability}

For $N \geq 1$ let $\left(a_{i, j}^{N}\right),\left(B_{i}^{N}\right)$ and $\left(\beta_{i, j}\right)$ be kinetic coefficients satisfying (6), (7) and (8), respectively. We further assume that they satisfy (9) uniformly with respect to $N \geq 1$, that is,

$$
\lim _{j \rightarrow+\infty} \sup _{N \geq 1} \frac{a_{i, j}^{N}}{j}=\lim _{j \rightarrow+\infty} \sup _{N \geq 1} \frac{B_{i+j}^{N} \beta_{i+j, i}}{i+j}=0 .
$$

and there holds

$$
\lim _{N \rightarrow+\infty} a_{i, j}^{N}=a_{i, j} \text { and } \lim _{N \rightarrow+\infty} B_{i}^{N}=B_{i}, \quad i, j \geq 1 .
$$

We also fix a sequence $\left(c^{i n, N}\right)_{N}$ in $X_{1}^{+}$. The stability result then reads as follows.

Theorem 3 For each $N \geq 1$, let $c^{N}=\left(c_{i}^{N}\right)_{i}$ be a weak solution to (1)-(3) with kinetic coefficients $\left(a_{i, j}^{N}\right),\left(B_{i}^{N}\right),\left(\beta_{i, j}\right)$ and initial datum $c^{\text {in,N }}$. Assume further that there is $c^{i n} \in X_{1}^{+}$such that

$$
c^{i n, N} \rightarrow c^{i n} \quad \text { in } X_{1} .
$$

Then there are a subsequence $\left(c^{N_{k}}\right)_{k}$ of $\left(c^{N}\right)$ and a function $c \in L^{\infty}\left(0,+\infty ; X_{1}^{+}\right)$ such that

$$
c_{i}^{N_{k}} \longrightarrow c_{i} \text { in } L^{1}((0, T) \times \Omega)
$$

for every $T \in(0,+\infty)$ and $i \geq 1$ and $c$ is a weak solution to (1)-(3) with kinetic coefficients $\left(a_{i, j}\right),\left(B_{i}\right),\left(\beta_{i, j}\right)$ and initial datum $c^{i n}$.

Proof. Let $T \in(0,+\infty)$ and put $\Omega_{T}=(0, T) \times \Omega$. We also define $Q_{i}^{N}($.$) ,$ $L_{i}^{N}($.$) and Q_{k, i}^{N}($.$) for k \in\{1, \ldots, 4\}, i \geq 1$ and $N \geq 1$ as $Q_{i}(),. L_{i}($.$) and$ $Q_{k, i}($.$) with \left(a_{i, j}^{N}\right),\left(B_{i}^{N}\right)$ instead of $\left(a_{i, j}\right),\left(B_{i}\right)$. Notice that it follows from (17) and (18) that, for each $i \geq 1$, there is a constant $C_{i}$ such that, for $N \geq 1$ and $j \geq 1$,

$$
\frac{a_{i, j}^{N}}{j}+B_{i}^{N}+\frac{B_{i+j}^{N} \beta_{i+j, i}}{i+j} \leq C_{i}
$$

- Step 1 : Boundedness of the reaction terms in $L^{1}\left(\Omega_{T}\right)$.

Observe first that (19) guarantees that

$$
\sup _{t \in[0,+\infty)}\left\|c^{N}(t)\right\|_{1} \leq C_{0}, \quad N \geq 1
$$


for some constant $C_{0}$. Next let $i \geq 1$. It readily follows from (21) and (22) that

$$
\left|Q_{2, i}^{N}\left(c^{N}\right)\right|_{L^{1}\left(\Omega_{T}\right)}+\left|Q_{4, i}^{N}\left(c^{N}\right)\right|_{L^{1}\left(\Omega_{T}\right)} \leq C_{i}
$$

We next claim that there also holds

$$
\left|Q_{1, i}^{N}\left(c^{N}\right)\right|_{L^{1}\left(\Omega_{T}\right)}+\left|Q_{3, i}^{N}\left(c^{N}\right)\right|_{L^{1}\left(\Omega_{T}\right)} \leq C_{i}
$$

for each $i \geq 1$ and we argue by induction on $i \geq 1$. Indeed let us first consider the case $i=1$. Then $Q_{1,1}^{N}\left(c^{N}\right)=Q_{2,1}^{N}\left(c^{N}\right)=0$ and it follows from (15) after integration over $\Omega_{T}$ and the non-negativity of $c_{1}^{N}$ that

$$
\int_{0}^{T} \int_{\Omega} Q_{3,1}^{N}\left(c^{N}\right) d x d s \leq\left|c_{1}^{i n, N}\right|_{L^{1}(\Omega)}+\left|Q_{4,1}^{N}\left(c^{N}\right)\right|_{L^{1}\left(\Omega_{T}\right)},
$$

and the right-hand side of the above inequality is bounded uniformly with respect to $N$ by (19) and (23). Therefore the claim (24) is true for $i=1$. Fix now $i \geq 1$ and assume that the claim (24) holds true for each $j \in\{1, \ldots, i\}$. Since

$$
Q_{1, i+1}^{N}\left(c^{N}\right) \leq \sum_{j=1}^{i} Q_{3, j}^{N}\left(c^{N}\right),
$$

the induction assumption entails that $\left(Q_{1, i+1}^{N}\left(c^{N}\right)\right)$ is bounded in $L^{1}\left(\Omega_{T}\right)$ uniformly with respect to $N$. It next follows from (15) after integration over $\Omega_{T}$ and the non-negativity of $c_{i+1}^{N}$ and $B_{i+1}^{N}$ that

$\int_{0}^{T} \int_{\Omega} Q_{3, i+1}^{N}\left(c^{N}\right) d x d s \leq\left|c_{i+1}^{i n, N}\right|_{L^{1}(\Omega)}+\left|Q_{1, i+1}^{N}\left(c^{N}\right)\right|_{L^{1}\left(\Omega_{T}\right)}+\left|Q_{4, i+1}^{N}\left(c^{N}\right)\right|_{L^{1}\left(\Omega_{T}\right)}$,

and the right-hand side of the above inequality is bounded uniformly with respect to $N$ by (19), (23). Consequently, the claim (24) holds true for each $j \in\{1, \ldots, i+1\}$ and the proof of (24) is complete.

- Step 2 : componentwise strong compactness in $L^{1}\left(\Omega_{T}\right)$.

Fix $i \geq 1$. It follows from (19), (23) and (24) that, for each $N \geq 1, c_{i}^{N}$ is a solution to a linear heat equation with initial data and right-hand sides uniformly bounded in $L^{1}(\Omega)$ and $L^{1}\left(\Omega_{T}\right)$, respectively. Since $A_{1}$ generates a positive, compact and analytic semigroup in $L^{1}(\Omega)$ by [1] we are thus in a position to apply the compactness results of $[5,4]$ and conclude that $\left(c_{i}^{N}\right)_{N}$ is relatively strongly compact in $L^{1}\left(\Omega_{T}\right)$. Therefore there are a subsequence 
of $\left(c^{N}\right)$ (not relabeled) and a sequence of functions $c=\left(c_{i}\right)_{i \geq 1}$ such that, for each $i \geq 1$,

$$
c_{i}^{N} \longrightarrow c_{i} \text { in } L^{1}\left(\Omega_{T}\right) \text { and a.e. in } \Omega_{T} \text {. }
$$

A first consequence of (26), (22) and the non-negativity of $c_{i}^{N}$ is that

$$
c \in L^{\infty}\left(0, T ; X_{1}^{+}\right) \quad \text { with } \sup _{t \in[0, T)}\|c(t)\|_{1} \leq C_{0} .
$$

It next follows from (26), (17) and (18) that, for each $i \geq 1$, there holds

$$
\left\{\begin{array}{c}
Q_{2, i}^{N}\left(c^{N}\right) \longrightarrow Q_{2, i}(c) \text { in } L^{1}\left(\Omega_{T}\right), \\
Q_{4, i}^{N}\left(c^{N}\right) \longrightarrow Q_{4, i}(c) \text { in } L^{1}\left(\Omega_{T}\right), \\
L_{i}^{N}\left(c^{N}\right) \longrightarrow L_{i}(c) \text { in } L^{1}\left(\Omega_{T}\right) .
\end{array}\right.
$$

- Step 3 : weak compactness of the coagulation terms in $L^{1}\left(\Omega_{T}\right)$.

Let $i \geq 1$ and $r>0$. For $N \geq 1$ we multiply the $i$-th equation of (15) by $\operatorname{sign}_{+}\left(c_{i}^{N}-r\right)$ and integrate over $\Omega_{T}$ to obtain

$$
\text { (28) } \begin{aligned}
\int_{\left\{c_{i}^{N}>r\right\}} c_{i}^{N} L_{i}^{N}\left(c^{N}\right) d x d s & \leq \int_{\Omega}\left(c_{i}^{i n, N}-r\right)_{+} d x \\
& +\int_{\left\{c_{i}^{N}>r\right\}}\left(Q_{1, i}^{N}\left(c^{N}\right)+Q_{4, i}^{N}\left(c^{N}\right)\right) d x d s .
\end{aligned}
$$

In order to justify (28) one may proceed in the following classical way : given a smooth non-negative, non-decreasing, convex and Lipschitz continuous function $\varphi: \mathbb{R} \rightarrow \mathbb{R}$ and the solution $u$ to

$$
\partial_{t} u-\Delta_{x} u+f=g, \quad \partial_{n} u=0, \quad u(0)=u_{0},
$$

with non-negative and smooth data $u_{0}, f$ and $g$ there holds

$$
\begin{gathered}
\int_{\Omega} \varphi(u(T)) d x+\int_{0}^{T} \int_{\Omega} \varphi^{\prime \prime}(u)|\nabla u|^{2} d x d s+\int_{0}^{T} \int_{\Omega} \varphi^{\prime}(u) f d x d s \\
=\int_{\Omega} \varphi\left(u_{0}\right) d x+\int_{0}^{T} \int_{\Omega} \varphi^{\prime}(u) g d x d s .
\end{gathered}
$$

The first two terms of the left-hand side of the above equality being nonnegative thanks to the properties of $\varphi$ it is easily seen that

$$
\int_{0}^{T} \int_{\Omega} \varphi^{\prime}(u) f d x d s \leq \int_{\Omega} \varphi\left(u_{0}\right) d x+\int_{0}^{T} \int_{\Omega} \varphi^{\prime}(u) g d x d s
$$


also holds true for any solution $u$ corresponding to non-negative functions $u_{0} \in L^{1}(\Omega)$ and $f, g \in L^{1}\left(\Omega_{T}\right)$. We may finally approximate the function $\xi \mapsto(\xi-r)_{+}$by a sequence $\left(\varphi_{\varepsilon}\right)$ of functions fulfilling the above requirements and obtain (28) in the limit $\varepsilon \rightarrow 0$.

Owing to (22) we have

$$
\text { meas }\left\{(s, x) \in \Omega_{T}, c_{i}^{N}(s, x)>r\right\} \leq \frac{C_{0} T}{r},
$$

from which we deduce that

$$
\text { (29) } \begin{aligned}
& \sup _{N \geq 1} \int_{\left\{c_{i}^{N}>r\right\}} c_{i}^{N} L_{i}^{N}\left(c^{N}\right) d x d s \\
\leq & \sup _{N \geq 1} \int_{\Omega}\left(c_{i}^{i n, N}-r\right)_{+} d x+\sup _{|E| \leq C_{0} T / r} \sup _{N} \int_{E}\left(Q_{1, i}^{N}\left(c^{N}\right)+Q_{4, i}^{N}\left(c^{N}\right)\right) d x d s .
\end{aligned}
$$

We then claim that

$$
\begin{aligned}
& \text { the sequences }\left(Q_{1, i}^{N}\left(c^{N}\right)\right)_{N} \text { and }\left(Q_{3, i}^{N}\left(c^{N}\right)\right)_{N} \text { are weakly } \\
& \text { compact in } L^{1}\left(\Omega_{T}\right) \text { for each } i \geq 1 \text {. }
\end{aligned}
$$

Indeed we argue by induction and first consider the case $i=1$. Since $Q_{1,1}^{N}\left(c^{N}\right)=0$ we infer from (29), (19) and (27) that

$$
\lim _{r \rightarrow+\infty} \sup _{N \geq 1} \int_{\left\{c_{1}^{N}>r\right\}} c_{1}^{N} L_{1}^{N}\left(c^{N}\right) d x d s=0 .
$$

Consequently, if $E$ is a measurable subset of $\Omega_{T}$ and $r>0$,

$$
\begin{aligned}
\sup _{N \geq 1} \int_{E} c_{1}^{N} L_{1}^{N}\left(c^{N}\right) d x d s \leq & r \sup _{N \geq 1} \int_{\left\{E \cap\left\{c_{1}^{N} \leq r\right\}\right\}} L_{1}^{N}\left(c^{N}\right) d x d s \\
& +\sup _{N \geq 1} \int_{\left\{c_{1}^{N}>r\right\}} c_{1}^{N} L_{1}^{N}\left(c^{N}\right) d x d s .
\end{aligned}
$$

Owing to the compactness $(27)$ of $\left(L_{1}^{N}\left(c^{N}\right)\right)$ in $L^{1}\left(\Omega_{T}\right)$ we may pass to the limit as $|E| \rightarrow 0$ in the above inequality to obtain

$$
\limsup _{|E| \rightarrow 0} \sup _{N \geq 1} \int_{E} c_{1}^{N} L_{1}^{N}\left(c^{N}\right) d x d s \leq \sup _{N \geq 1} \int_{\left\{c_{1}^{N}>r\right\}} c_{1}^{N} L_{1}^{N}\left(c^{N}\right) d x d s .
$$

The above inequality being valid for every $r>0$ the claim (30) for $i=1$ follows at once from (31), the above inequality and the Dunford-Pettis theorem. 
Let us now consider $i \geq 1$ and assume that the claim (30) holds true for each $j \in\{1, \ldots, i\}$. We readily infer from the induction assumption and (25) that $\left(Q_{1, i+1}^{N}\left(c^{N}\right)\right)$ is weakly compact in $L^{1}\left(\Omega_{T}\right)$. This last fact, together with (29), (19) and (27) yield that

$$
\lim _{r \rightarrow+\infty} \sup _{N \geq 1} \int_{\left\{c_{i+1}^{N}>r\right\}} c_{i+1}^{N} L_{i+1}^{N}\left(c^{N}\right) d x d s=0 .
$$

We next proceed as before to conclude that $\left(Q_{3, i+1}^{N}\left(c^{N}\right)\right)$ is weakly compact in $L^{1}\left(\Omega_{T}\right)$ and the proof of $(30)$ is complete.

Now, fix $i \geq 1$ and observe that (17), (18) and (26) ensure that the sequences $\left(Q_{1, i}^{N}\left(c^{N}\right)\right)$ and $\left(Q_{3, i}^{N}\left(c^{N}\right)\right)$ converge almost everywhere in $\Omega_{T}$ towards $Q_{1, i}(c)$ and $Q_{3, i}(c)$, respectively. Recalling (30) we infer from the Vitali theorem that

$$
\left\{\begin{array}{l}
Q_{1, i}^{N}\left(c^{N}\right) \longrightarrow Q_{1, i}(c) \text { in } L^{1}\left(\Omega_{T}\right), \\
Q_{3, i}^{N}\left(c^{N}\right) \longrightarrow Q_{3, i}(c) \text { in } L^{1}\left(\Omega_{T}\right) .
\end{array}\right.
$$

Owing to (19), (27) and (32) it is straightforward to pass to the limit in the mild formulation (15) of (1) and conclude that $c$ is a weak solution to (1)-(3) (observe that we may take $T$ to be an arbitrary large integer and perform countably many successive extractions).

\section{Existence}

In this section we prove Theorem 2. For that purpose we first introduce a sequence of approximating problems which are obtained by truncating (1). More precisely, for $N \geq 3$ we put

$$
\begin{gathered}
a_{i, j}^{N}=\left\{\begin{array}{ccc}
a_{i, j}+1 / N & \text { if } & \max (i, j) \leq N, \\
0 & \text { if } \quad & \max (i, j)>N,
\end{array}\right. \\
B_{i}^{N}=\left\{\begin{array}{ccc}
B_{i} & \text { if } & i \leq N, \\
0 & \text { if } & i>N,
\end{array}\right.
\end{gathered}
$$

We also put

$$
c_{i}^{i n, N}=\left\{\begin{array}{ccc}
\min \left(c_{i}^{i n}, N\right) & \text { if } & i \leq N \\
0 & \text { if } & i>N
\end{array}\right.
$$


We next consider the following system of $2 N$ reaction-diffusion equations

$$
\begin{aligned}
\partial_{t} c_{i}^{N}-d_{i} \Delta_{x} c_{i}^{N} & =Q_{i}^{N}\left(c^{N}\right) \quad \text { in }(0,+\infty) \times \Omega, \\
\partial_{n} c_{i}^{N} & =0 \text { on }(0,+\infty) \times \partial \Omega, \\
c_{i}^{N}(0) & =c_{i}^{i n, N} \text { in } \Omega,
\end{aligned}
$$

for $i \in\{1, \ldots, 2 N\}$, where we have set $c^{N}=\left(c_{i}^{N}\right)_{i \geq 1}$ with $c_{i}^{N} \equiv 0$ for $i>2 N$ and $Q_{i}^{N}($.$) is defined as Q_{i}($.$) with \left(a_{i, j}^{N}\right),\left(B_{i}^{N}\right)$ instead of $\left(a_{i, j}\right),\left(B_{i}\right)$. We may then proceed as in [24, Lemma 2.2] to show that (36)-(38) has a unique solution $c^{N}$ on $[0,+\infty)$ with bounded components and such that

$$
\sup _{t \in[0,+\infty)}\left\|c^{N}(t)\right\|_{1} \leq\left\|c^{i n}\right\|_{1}, \quad N \geq 1
$$

Owing to (9), (33), (34), (35) and (39) it is straighforward to check that the sequence $\left(c^{N}\right)$ fulfils the requirements of Theorem 3 from which Theorem 2 readily follows, taking also into account that (39) warrants that (14) holds true.

\section{Acknowledgments}

We thank the referee for helpful suggestions to improve the clarity of the paper.

\section{References}

[1] Amann, H.: Dual semigroups and second order linear elliptic boundary value problems. Israel J. Math. 45 (1983), 225-254.

[2] Amann, H.: Coagulation-fragmentation processes. Arch. Rational Mech. Anal. 151 (2000), 339-366.

[3] Ball, J.M. AND CARR, J.: The discrete coagulation-fragmentation equations : existence, uniqueness, and density conservation. J. Statist. Phys. 61 (1990), 203-234.

[4] Baras, P.: Compacité de l'opérateur $f \mapsto u$ solution d'une équation non linéaire $(d u / d t)+A u \ni f$. C. R. Acad. Sci. Paris Sér. I Math. 286 (1978), $1113-1116$.

[5] Baras, P. Hassan, J.C. And Véron, L.: Compacité de l'opérateur définissant la solution d'une équation d'évolution non homogène. $C . R$. Acad. Sci. Paris Sér. I Math. 284 (1977), 799-802.

[6] Bénilan, Ph. and Wrzosek, D.: On a infinite system of reactiondiffusion equations. Adv. Math. Sci. Appl. 7 (1997), 349-364. 
[7] Chae, D. And DubovskiI , P.B.: Existence and uniqueness for spatially inhomogeneous coagulation equation with sources and effluxes. Z. Angew. Math. Phys. 46 (1995), 580-594.

[8] Collet, J.F. And Poupaud, F.: Existence of solutions to coagulationfragmentation systems with diffusion. Transport Theory Statist. Phys. 25 (1996), 503-513.

[9] Costa, F.P. DA: Existence and uniqueness of density conserving solutions to the coagulation-fragmentation equations with strong fragmentation. J. Math. Anal. Appl. 192 (1995), 892-914.

[10] Drake, R.L.: A general mathematical survey of the coagulation equation, in Topics in Current Aerosol Research (part 2). International Reviews in Aerosol Physics and Chemistry, Pergamon Press, Oxford, 1972, 203-376.

[11] Guiaş, F.: Convergence properties of a stochastic model for coagulationfragmentation processes with diffusion. Stochastic Anal. Appl. 19 (2001), $245-278$.

[12] Herrero, M.A., Velázquez, J.J.L. And Wrzosek, D.: Sol-gel transition in a coagulation-diffusion model. Phys. D 141 (2000), 221-247.

[13] JEON, I.: Existence of gelling solutions for coagulation-fragmentation equations. Comm. Math. Phys. 194 (1998), 541-567.

[14] Laurençot, Ph. And Wrzosek, D.: Fragmentation-diffusion model. Existence of solutions and asymptotic behaviour. Proc. Roy. Soc. Edinburgh Sect. A 128 (1998), 759-774.

[15] Laurençot, Ph. And Wrzosek, D.: The Becker-Döring model with diffusion. I. Basic properties of solutions. Colloq. Math. 75 (1998), 245-269.

[16] McGrady, E.D. And ZifF, R.M.: "Shattering" transition in fragmentation. Phys. Rev. Lett. 58 (1987), 892-895.

[17] Perelson, A.S. and Samsel, R.W.: Kinetics of red blood cell aggregation : an example of geometric polymerization, in Kinetics of Aggregation and Gelation. F. Family and D.P. Landau (eds.), Elsevier, 1984.

[18] Safronov, V.S.: Evolution of the Protoplanetary Cloud and Formation of the Earth and the Planets, Israel Program for Scientific Translations Ltd., Jerusalem, 1972.

[19] Simons, S. And Simpson, D.R.: The effect of particle coagulation on the diffusive relaxation of a spatially inhomogeneous aerosol. J. Phys. A 21 (1988), 3523-3536.

[20] Slemrod, M.: Coagulation-diffusion systems : derivation and existence of solutions for the diffuse interface structure equations. Phys. D 46 (1990), 351-366.

[21] Smoluchowski, M.: Drei Vorträge über Diffusion, Brownsche Molekularbewegung und Koagulation von Kolloidteilchen. Physik. Zeitschr. 17 (1916), 557-599. 
[22] Smoluchowski, M.: Versuch einer mathematischen Theorie der Koagulationskinetik kolloider Lösungen. Zeitschrift f. physik. Chemie 92 (1917), 129-168.

[23] Spouge, J.L.: An existence theorem for the discrete coagulationfragmentation equations. Math. Proc. Cambridge Philos. Soc. 96 (1984), 351-357.

[24] Wrzosek, D.: Existence of solutions for the discrete coagulationfragmentation model with diffusion. Topol. Methods Nonlinear Anal. 9 (1997), 279-296.

[25] Wrzosek, D.: Mass-conserving solutions to the discrete coagulationfragmentation model with diffusion. Nonlinear Anal. 49 (2002), 297-314.

Recibido: 21 de marzo de 2001

Revisado: 12 de junio de 2001

Philippe Laurençot Mathématiques pour l'Industrie et la Physique

UMR CNRS 5640

Université Paul Sabatier - Toulouse 3 118 route de Narbonne F-31062 Toulouse cedex 4, France laurencot@mip.ups-tlse.fr

Stéphane Mischler Laboratoire de Mathématiques Appliquées Université de Versailles - Saint Quentin 45 avenue des Etats-Unis F-78035 Versailles, France mischler@math.uvsq.fr 